\title{
Avaliação de Máquinas Preemptíveis nos Provedores de Nuvem Pública Amazon e Google
}

\author{
Jonas P. Soares, Aletéia P. F. de Araújo \\ ${ }^{1}$ Departamento de Ciência da Computação - Universidade de Brasília (UnB) \\ Brasília - DF - Brazil \\ \{pradojonas64, aleteia.araujo\}@gmail.com
}

\begin{abstract}
In the current diversity of cloud computing market, choosing the right service and provider becomes a non-trivial challenge for users. In this context, this paper proposes a comparative analysis on preemptible machines offered as a service by public cloud providers. For that goal, experimental tests are executed in instances offered by the providers, using benchmarks. The work concludes, from the results of cost and performance obtained, which instances, providers, and regions are best suited for benchmarked workloads.
\end{abstract}

Resumo. Com a diversidade de serviços do mercado de nuvem computacional, a escolha do serviço e do provedor mais adequados consiste em um desafio não trivial para os usuários. Nesse contexto, este trabalho propõe uma análise comparativa sobre máquinas preemptíveis oferecidas por provedores de nuvem pública, as quais podem ter a execução finalizada em situações nas quais seus recursos computacionais são necessários em outras tarefas do provedor do serviço. Para isso, são executados testes experimentais em instâncias oferecidas pelos provedores, utilizando benchmarks da literatura. O trabalho conclui, a partir dos resultados de custo e de performance obtidos, quais instâncias, provedores e regiões são mais indicados para cargas de trabalho similares aos benchmarks executados.

\section{Introdução}

Identificando a possibilidade de diminuir perdas causadas pelas desvantagens do modelo de instâncias dedicadas, e diversificar seus catálogo de serviços, alguns provedores de nuvem pública lançaram projetos de instâncias preemptíveis [Amazon 2009] [Google 2015]. Nesse modelo, as instâncias podem ser finalizadas em situações onde seus recursos computacionais são necessários em outras tarefas do provedor do serviço. Além disso, a confiabilidade oferecida por essas instâncias é diferente do modelo dedicado, o qual oferece garantias de serviço por meio de SLAs [Zhang et al. 2011]. Em contrapartida, a execução de máquinas preemptíveis custa apenas uma fração do valor cobrado por máquinas dedicadas com a mesma configuração. Desta maneira, o modelo se torna interesssante para o mercado consumidor, e pode gerar receita ao provedor a partir de recursos previamente ociosos [Subramanya et al. 2015].

Nesse contexto, este trabalho propõe uma análise comparativa sobre máquinas preemptíveis oferecidas por provedores de nuvem pública, especificamente sobre os provedores Amazon ${ }^{1}$ e Google ${ }^{2}$ - provedores líderes de mercado.

\footnotetext{
${ }^{1}$ https://aws.amazon.com/

${ }^{2}$ https://cloud.google.com/
} 


\section{Provedores avaliados}

Diante desse contexto, apenas os provedores de nuvem Amazon e Google tiveram suas máquinas incluídas no escopo deste trabalho, cujos serviços de máquinas preemptíveis são apresentados nesta seção.

\subsection{Amazon Web Services}

As máquinas preemptíveis oferecidas pela Amazon Web Services, chamadas Instâncias Spot, têm sua disponibilidade definida por um mercado de leilões, de maneira que os preços das instâncias (chamados preços Spot) são ajustados dinamicamente com base na oferta e na demanda de cada tipo de instância. Uma vez que um usuário ofereça um valor superior ao preço Spot vigente, ele ganha acesso às máquinas virtuais em questão - possibilitando uma economia monetária em períodos de baixa demanda pelas instâncias [Yi et al. 2010]. Em suma, o modelo Spot faz com que o preço praticado pelo provedor se aproxime do ponto de equilíbrio de mercado mais consistentemente que no modelo dedicado, de precificação fixa. Além disso, as instâncias dessa categoria já são amplamente exploradas pela literatura [Zhang et al. 2011, Subramanya et al. 2015, Taifi et al. 2011, Yi et al. 2010].

\subsection{Google Compute Engine}

As instâncias preemptíveis da plataforma Google Compute Engine operam em um modelo de precificação fixo similar às máquinas de uso dedicado, ou seja, não operam com preços de leilão. Nesse modelo, essas instâncias possuem preço por hora de execução consideravelmente mais baixo, mas contam com algumas limitações. Primeiramente, elas não são contempladas por nenhum SLA do provedor, de maneira que não há compensação ao usuário por períodos de indisponibilidade das instâncias. No que se refere a interrupções, essas máquinas sempre serão preemptadas após 24 horas contínuas de execução. Além dessa interrupção após 24 horas, instâncias dessa categoria podem estar indisponíveis ou ser preemptadas após intervalos de tempo menores, devido ao esgotamento de recursos do GCE ou outros eventos do sistema [Google 2015].

\section{Metodologia}

Segundo Li et al. [Li et al. 2011], é importante que a comparação entre serviços de nuvem seja realizada por meio de métricas que desconsiderem detalhes de implementação de cada provedor, e tenham foco no desempenho de ponta a ponta dos serviços. O trabalho aqui proposto explora, especificamente sob a perspectiva de máquinas preemptíveis, quatro das métricas propostas por Li: tempo de execução do benchmark, custo monetário por execução do benchmark, taxa de transferência para dispositivos de armazenamento, e custo com operações de leitura e de escrita nesses dispositivos [Li et al. 2011]. Assim, com o objetivo de avaliar a execução de cargas de trabalho com diferentes comportamentos, foram executados testes com os benchmarks SPECjvm2008 [SPEC 2008] e Sysbench fileio [Kopytov 2004], os quais são caracterizados por um processamento intensivo e por um grande volume de operações de leitura e escrita, respectivamente.

A escolha dos provedores contemplados nos testes foi tomada com base no quadrante mágico divulgado pela companhia Gartner, referente ao ano de 2018 [Shiff 2018]. Segundo análise da companhia, o provedor Amazon manteve sua liderança no mercado 
Tabela 1. Máquinas contempladas por execuções SPECjvm2008.

\begin{tabular}{|c|c|c|c|c|c|}
\hline \multicolumn{3}{|c|}{ Google } & \multicolumn{3}{c|}{ Amazon } \\
\hline Instância & vCPUs & RAM (GB) & Instância & vCPUs & RAM (GB) \\
\hline small & $1^{3}$ & 1,7 & t3small & 2 & 2 \\
\hline standardHighCPU & 1 & 1,5 & t3medium & 2 & 4 \\
\hline standard & 1 & 3,75 & t3large & 2 & 8 \\
\hline doubleHighCPU & 2 & 1,8 & t3xlarge & 4 & 16 \\
\hline double & 2 & 7,5 & c4large & 2 & 3,75 \\
\hline doubleHighMem & 2 & 13 & c5large & 2 & 4 \\
\hline quadrupleHighCPU & 3,6 & 22,5 & c5xlarge & 4 & 8 \\
\hline quadruple & 4 & 15 & c52xlarge & 8 & 16 \\
\hline octacoreHighCPU & 8 & 7,2 & - & - & - \\
\hline octacore & 8 & 30 & - & - & - \\
\hline
\end{tabular}

de IaaS, o que motivou sua inclusão no escopo deste trabalho. Como segundo provedor, o Google foi selecionado devido à sua recente inclusão no grupo de líderes. Ainda que apresente grande relevância segundo a análise pela Gartner, a Microsoft não foi contemplada por esta análise devido ao seu ingresso ainda muito recente no mercado de máquinas preemptíveis, datado de 2017 [McCrory 2018] - Amazon e Google trouxeram suas versões do serviço já em 2009 e 2015, respectivamente.

Definido o escopo do trabalho, foram executados testes em ambos os provedores, cujos resutltados são apresentados na seção a seguir. As máquinas Google e Amazon avaliadas nestes testes foram instanciadas nas regiões localizada em Carolina do Sul-USA e Ohio-USA, regiões recomendadas pelos provedores devido ao custo mais baixo dentre as disponíveis.

\section{Resultados}

\subsection{Benchmarks SPECjvm2008}

As configurações de instâncias contempladas pela análise do SPECjvm2008 são apresentadas na Tabela 1. Visando reduzir o número de variáveis da análise, apenas amostras de regiões us-east, localizadas no Leste dos Estados Unidos, foram consideradas nestes gráficos. Além disso, este trabalho contemplou exclusivamente máquinas de pequeno porte, com até 8 vCPUs.

Visando identificar instâncias dos provedores Google e Amazon que ofereçam menor custo e tempo de resposta na execução dos benchmarks SPECjvm2008, os gráficos apresentados nas Figuras 1 a 4 são baseados na simulação de uma carga de trabalho composta por um milhão de operações dos benchmarks avaliados, partindo do coeficiente de operações por minuto obtido pelas instâncias nos testes realizados. $\mathrm{O}$ eixo $X$ dessses gráficos apresenta o tempo de execução das cargas em horas, enquanto o eixo $Y$ descreve o custo em dólares para a execução das cargas.

Em leitura da Figura 1, a instância small apresenta uma nítida desvantagem em relação às demais máquinas Google avaliadas na simulação, com tempo de execução

\footnotetext{
${ }^{3}$ vCPU com núcleo compartilhado
} 

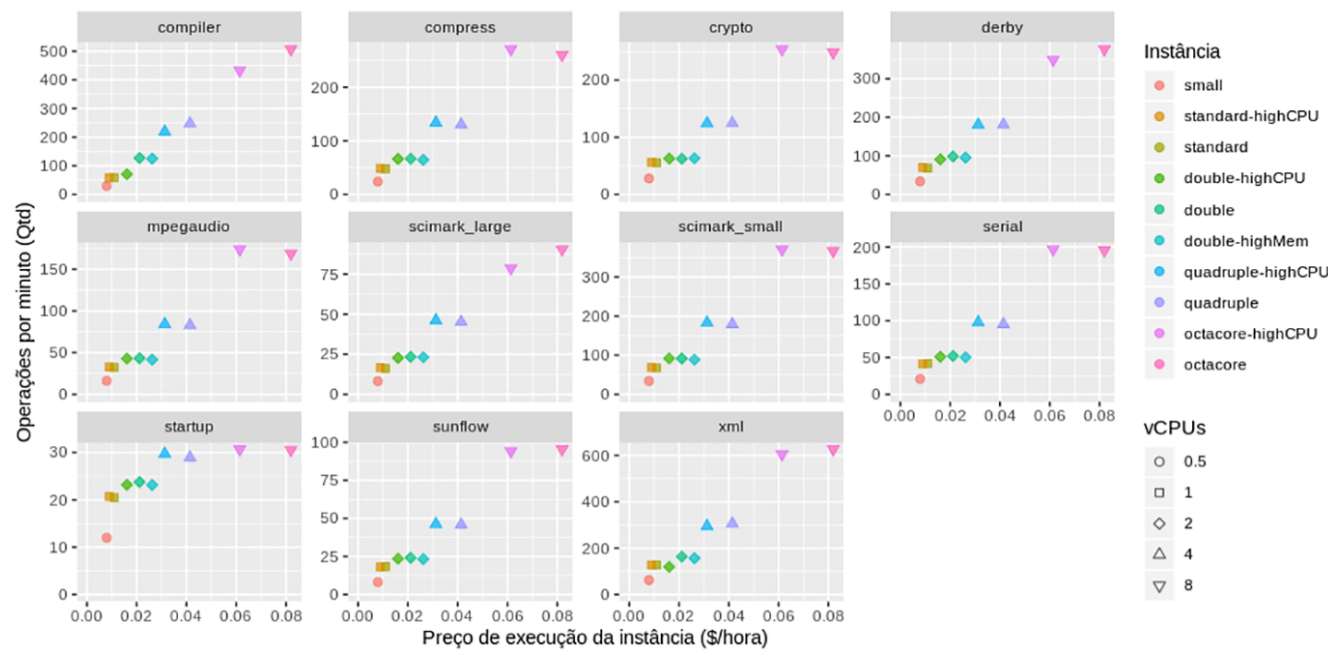

Figura 1. Desempenho SPECjvm2008 por benchmark incluindo instâncias High (Google).

muito superior em todos os benchmarks, e custo mais elevado em grande parte deles. Essa configuração caracteriza máquinas com vCPU compartilhada, de maneira que o recurso físico é compartilhado entre diversas instâncias small. Além disso, é possível observar que o desempenho dos benchmarks SPECjvm2008 é diretamente impactado pelo número de vCPUs da máquina virtual, e pouco sensível à memória RAM disponível - vide agrupamentos de amostras com mesmo número de vCPUs.

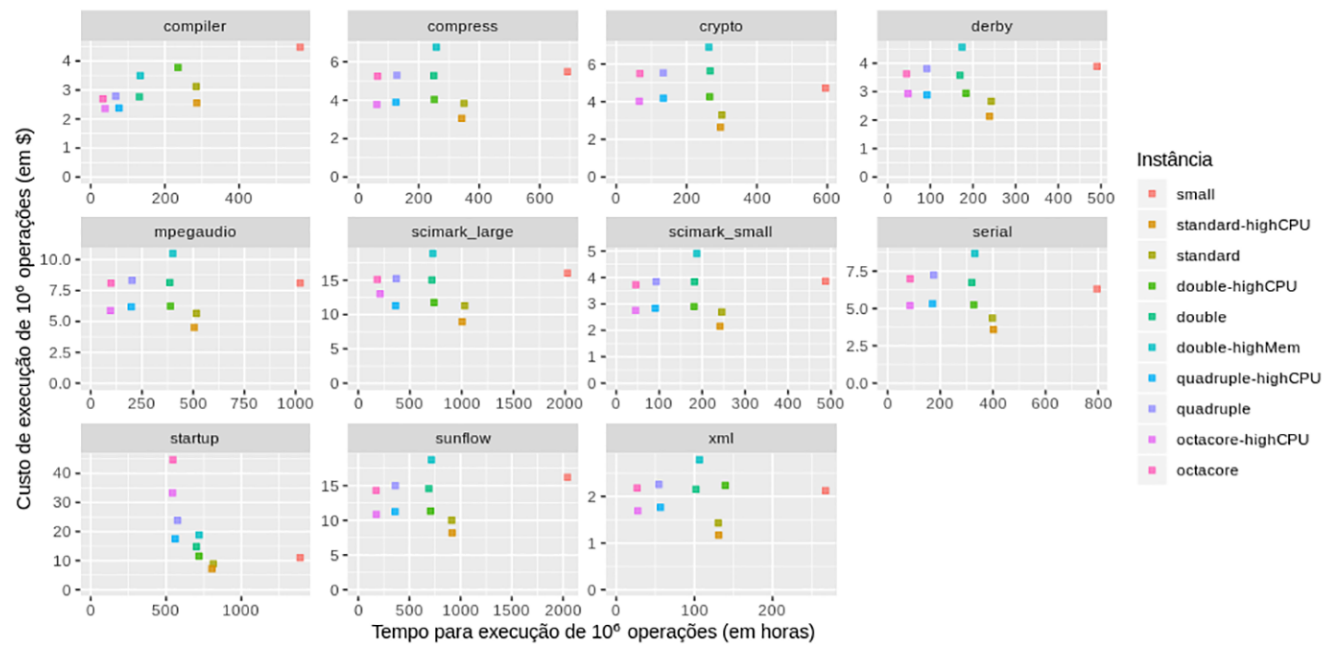

Figura 2. Execução de uma carga de trabalho SPECjvm2008 com um milhão de operações (Google).

Constatado que unidades de processamento consistem no principal gargalo do SPECjvm2008, foram executadas as instâncias que apresentassem os maiores índices de vCPU por RAM entre as disponibilizadas pela Amazon, as quais são compostas pela divisão do número de vCPUs pela memória RAM disponível (em GB). Assim, para este teste foram utilizadas máquinas de diferentes famílias (T3, C4, C5), cujas configurações foram listadas na Tabela 1.

Comparando os pontos do gráfico na Figura 2, que apresenta os resultados obtidos 
na simulação proposta em instâncias Amazon, é possível constatar que não há uma categoria de máquinas preemptíveis que apresente liderança absoluta de desempenho. Assim como observado entre as máquinas Google, a instância Amazon que possui custo mais baixo é diferente daquela que apresenta tempo de execução mínimo, indicando um tradeoff entre as duas métricas. Sob uma perspectiva financeira, a configuração $t 3 \mathrm{small}$ (em roxo) se mostra a mais econômica, enquanto a c52xlarge (em verde) desponta como a mais eficiente em tempo total de execução.

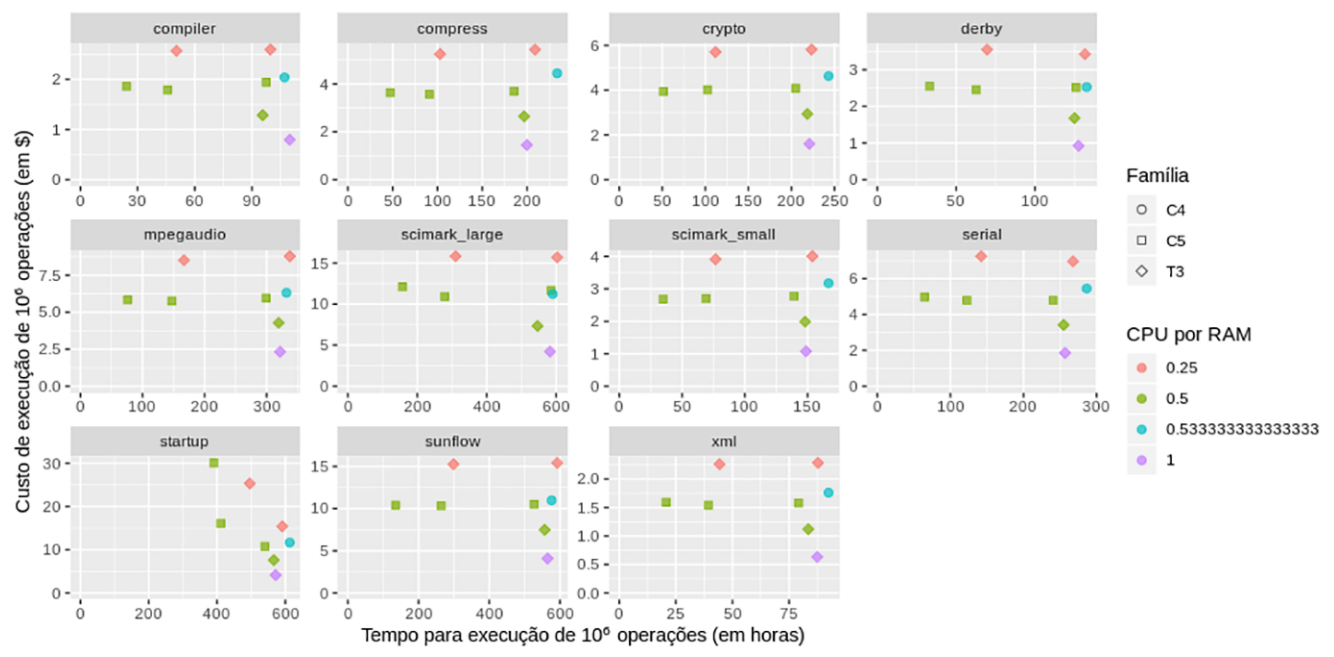

Figura 3. Execução de uma carga de trabalho SPECjvm2008 com um milhão de operações (Amazon).

Avaliadas as máquinas Google e Amazon separadamente, a Figura 4 condensa as amostras de ambos os provedores, e apresenta as amostras que registraram melhores preços e/ou tempo de resposta entre os testes realizados nesta seção. Logo, sob uma perspectiva que priorize o tempo de resposta, a instância octacoreHighCPU foi a que apresentou melhor performance dentre as máquinas Google, mas a instância Amazon c52xlarge registrou menor tempo de execução em todos os benchmarks, mantendo preços similares. Avaliando máquinas de categorias mais econômicas, a Amazon apresenta resultados ainda mais convidativos: suas instâncias $t 3$ small executaram a carga de trabalho em menos tempo e com economia de aproximadamente $40 \%$ em relação à instância Google mais econômica identificada (standardHighCPU). Diante do exposto, conclui-se que as instâncias preemptíveis Amazon EC2 apresentam melhor custo-benefício na execução de cargas de trabalho dos benchmarks SPECjvm2008 ou de aplicações semelhantes.

\subsection{Benchmark Sysbench fileio}

Enquanto o benchmark SPECjvm2008 conta com 11 processos de trabalho singulares, e produz resultados individuais para cada um deles, a execução do benchmark Sysbench em modo fileio produz um único coeficiente para análise e comparações. A Tabela 2 apresenta as principais informações sobre as máquinas contempladas pelos testes Sysbench fileio, os quais tiveram como base a simulação da carga de trabalho de um bilhão de operações do benchmark. Todas as instâncias foram equipadas com discos de $100 \mathrm{~GB}$ e configuradas com um domínio de teste com 80 GBs para leitura e escrita aleatórias de dados - valor esse escolhido para que caracterizasse um volume de dados muito superior à memória RAM disponível nas instâncias. 


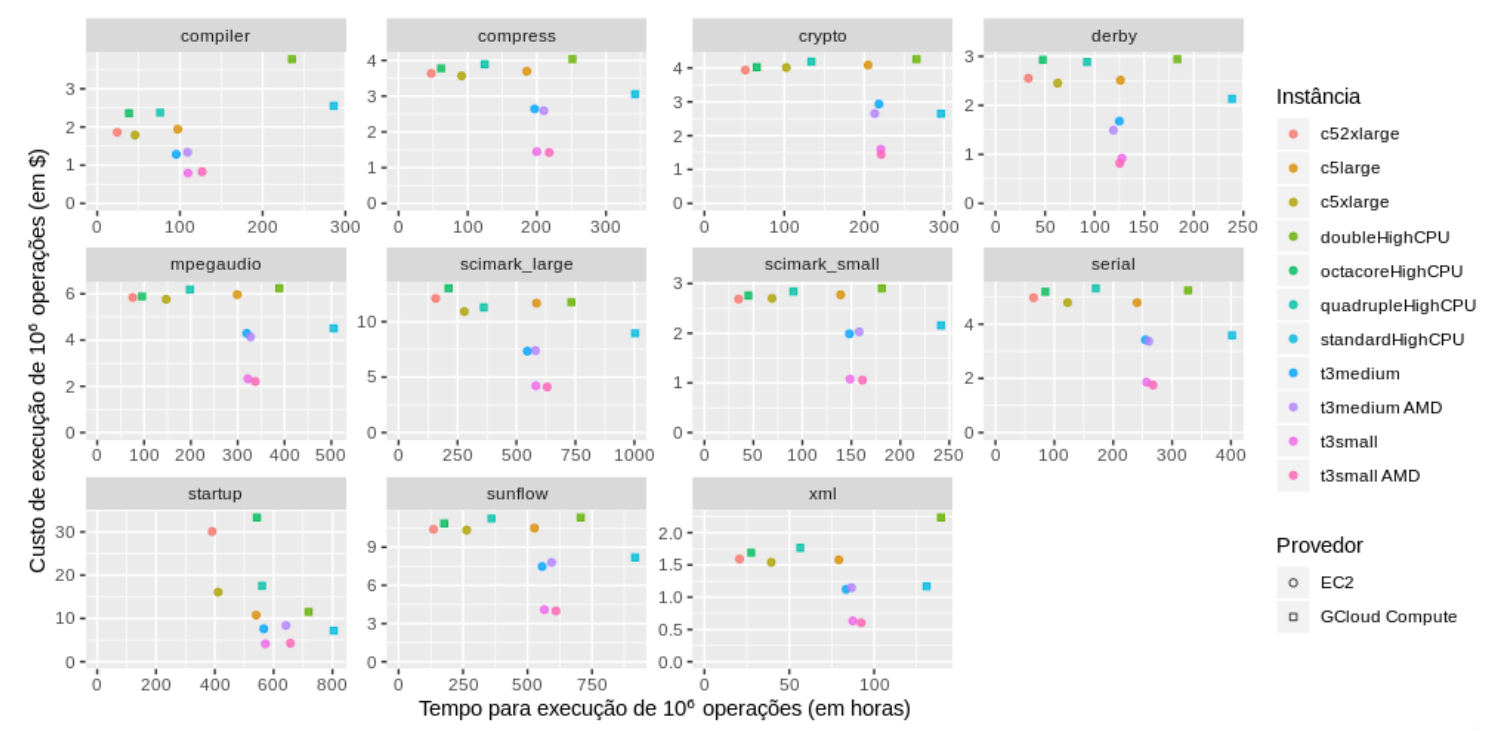

Figura 4. Execuções SPECjvm2008 nos provedores Google e Amazon.

Tabela 2. Instâncias contempladas por execuções Sysbench fileio.

\begin{tabular}{|c|c|c|c|c|}
\hline Instância & $\begin{array}{c}\text { Google } \\
\text { t3nano }\end{array}$ & $\begin{array}{c}\text { Google } \\
\text { t3small }\end{array}$ & $\begin{array}{c}\text { Amazon } \\
\text { small06 }\end{array}$ & $\begin{array}{c}\text { Amazon } \\
\text { standard }\end{array}$ \\
\hline vCPU & 2 & 2 & 0.5 & 1 \\
\hline RAM (GB) & 0.5 & 2 & 0.6 & 3.75 \\
\hline
\end{tabular}

Uma configuração personalizável durante a criação de instâncias preemptíveis é o dispositivo utilizado para armazenamento, alterando de um disco rígido (HDD) para uma unidade de estado sólido (SSD). Segundo a documentação da Amazon ${ }^{4}$, SSDs são equipamentos recomendados para cargas de trabalho com leitura e escrita de dados em grandes volumes ou intensiva, como é o caso do Sysbench fileio. Para investigar este comportamento, foram realizados testes em ambos os dispositivos.

Em leitura do gráfico apresentado na Figura 5, que apresenta os resultados obtidos pelas simulações, fica evidente o ganho econômico e performático obtido com os discos SSDs. quando comparados com os resultados de execuções HDD. Além disso, concluise que as máquinas preemptíveis do provedor Google equipadas com discos SSD são as mais recomendadas para execução de cargas de trabalho com fluxo intenso de leitura e de escrita, uma vez que apresentaram o menor custo e tempo de resposta dentre as execuções simuladas no benchmark Sysbench fileio.

\section{Conclusões e Trabalhos Futuros}

Este trabalho propôs uma avaliação dos serviços de máquinas preemptíveis oferecidos pelos provedores de nuvem pública Amazon e Google, com foco em analisar custo e performance a partir do interesse do usuário de usufruir de um serviço adequado pelo menor preço. Em relação aos testes com os benchmarks SPECjvm2008, as instâncias preemptíveis instanciadas pela Amazon apresentaram resultados com performance superior, e custo reduzido em diversas faixas de preço. Com isso, as máquinas preemptíveis

\footnotetext{
${ }^{4}$ https: / / aws.amazon.com/ebs/features/
} 


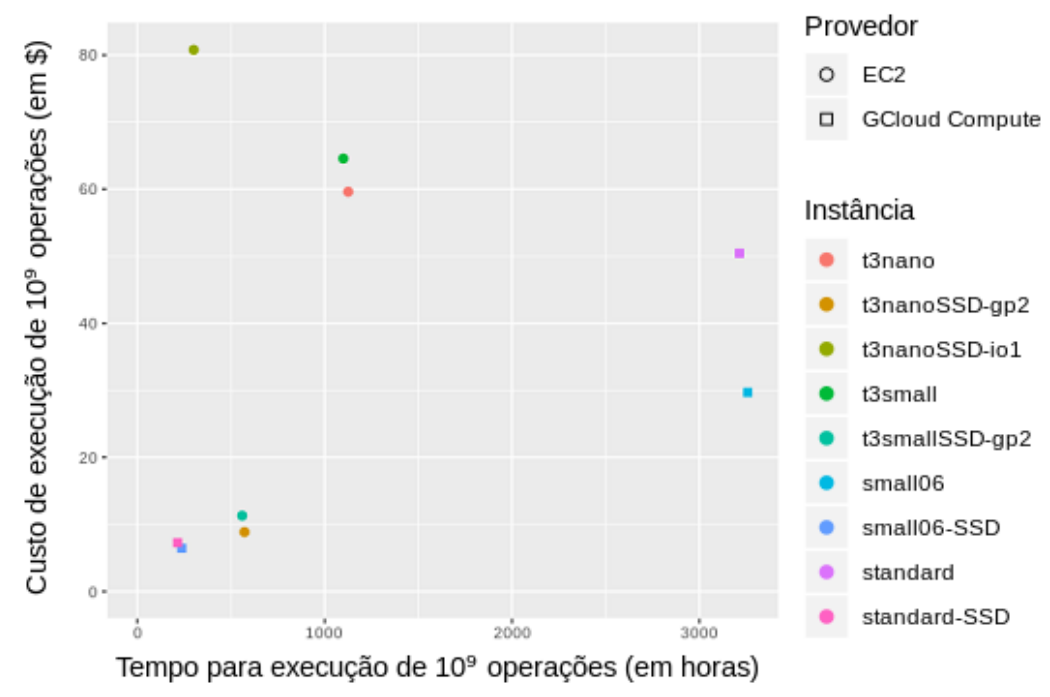

Figura 5. Execução de carga de trabalho Sysbench fileio incluindo instâncias equipadas com SSD.

da Amazon se mostraram mais indicadas para execução de cargas de trabalho com processamento intensivo, como o SPECjvm2008.

Analisando os resultados do benchmark Sysbench fileio, observou-se as categorias de máquinas mais indicadas para execução intensiva de operações I/O aleatórias são as de menor custo, sendo essas as famílias t3nano na Amazon e small06 no Google. Além disso, a execução do benchmark em instâncias equipadas com dispositivos SSD apresentou grande vantagem econômica em relação aos HDD. Comparando os provedores, conclui-se que as instâncias Google equipadas com SSD são as mais recomendadas para cargas de trabalho limitadas pela leitura e escrita aleatória, uma vez que se mostraram superiores em termos econômicos e de tempo de execução.

Quanto a usabilidade, as preempções diárias previstas pelas máquinas preemptíveis do Google podem trazer problemas relevantes para cliente do serviço, enquanto as instâncias Spot da Amazon têm sua execução garantida com maior grau de confiabilidade, caso o usuário esteja disposto a pagar mais caro quando necessário.

Para trabalhos futuros, sugere-se que sejam executados testes considerando outros provedores, como Microsoft Azure e Oracle Cloud. Além disso, é interessante avaliar o uso de GPUs em máquinas preemptíveis e seus consequentes impactos. Propõe-se também a realização de experimentos com outros benchmarks, cujas cargas de trabalho tenham caracteristicas diferentes do SPECjvm2008 e do Sysbench fileio. Alguns exemplos sugeridos são testes com aplicações diretamente impactadas pela memória RAM disponível, ou pela latência de comunicação entre um usuário cliente e um servidor em nuvem, onde a região geográfica da instância ganha maior relevância. Por fim, também sugere-se a análise comparativa entre máquinas preemptíveis e máquinas dedicadas, buscando maneiras de estimar o custo e o benefício vinculados à confiabilidade oferecida pelas máquinas sob demanda. 


\section{Referências}

Amazon (2009). Documentação da aws. https://docs.aws.amazon.com/ index.html. Acessado em: 2019-05-26.

Google (2015). Google compute engine service level agreement (sla). https: //cloud.google.com/compute/docs/machine-types?hl=pt-br \# sharedcore. Acessado em: 2019-06-15.

Kopytov, A. (2004). Sysbench manual. http://imysql.com/wp-content/ uploads/2014/10/sysbench-manual . pdf. Acessado em: 2019-06-21.

Li, A., Yang, X., Kandula, S., and Zhang, M. (2011). Comparing public-cloud providers. IEEE Internet Computing, 15(2):50-53.

McCrory, M. (2018). Announcing low-priority vms on scale sets now in public preview. https://azure.microsoft.com/pt-br/blog/ low-priority-scale-sets/. Acessado em: 2019-07-22.

Shiff, L. (2018). Gartner magic quadrant for cloud infrastructure as a service 2018. https://www.bmc.com/blogs/ gartner-magic-quadrant-cloud-iaas/. Acessado em: 2019-06-25.

SPEC (2008). Specjvm2008 user guide. https://www.spec.org/jvm2008/ docs/UserGuide.html. Acessado em: 2019-05-26.

Subramanya, S., Guo, T., Sharma, P., Irwin, D., and Shenoy, P. (2015). Spoton: a batch computing service for the spot market. In Proceedings of the sixth ACM symposium on cloud computing, pages 329-341. ACM.

Taifi, M., Shi, J. Y., and Khreishah, A. (2011). Spotmpi: a framework for auction-based hpc computing using amazon spot instances. In International Conference on Algorithms and Architectures for Parallel Processing, pages 109-120. Springer.

Yi, S., Kondo, D., and Andrzejak, A. (2010). Reducing costs of spot instances via checkpointing in the amazon elastic compute cloud. In 2010 IEEE 3rd International Conference on Cloud Computing, pages 236-243. IEEE.

Zhang, Q., Zhu, Q., and Boutaba, R. (2011). Dynamic resource allocation for spot markets in cloud computing environments. In 2011 Fourth IEEE International Conference on Utility and Cloud Computing, pages 178-185. IEEE. 